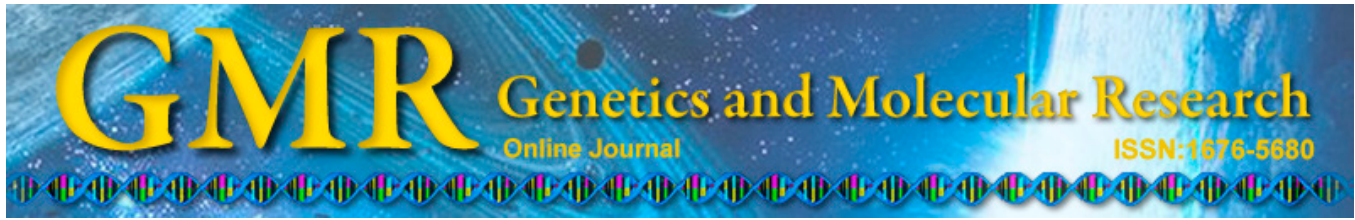

\title{
Characterization of $\mathrm{NF} 1$ frameshift mutations in pediatric patients with neurofibromatosis type I
}

J. Villa-Morales ${ }^{1,2}$, E. López-Muñoz ${ }^{3}$, D. Juárez-Melchor ${ }^{1}$,

N. García-Hernández ${ }^{1}$, F. Minauro-Sanmiguel ${ }^{1}$, J. Aguirre-Hernández ${ }^{4}$,

G. Gutiérrez-Iglesiass ${ }^{5}$ and D.J. Arenas-Aranda ${ }^{1}$

${ }^{1}$ Unidad de Investigación Médica en Genética Humana,

Hospital de Pediatría "Dr. Silvestre Frenk Freund",

Centro Médico Nacional Siglo XXI, Instituto Mexicano del Seguro Social, México, D.F., México

2Departamento de Genética, Hospital Infantil de México "Federico Gómez", México, D.F., México

${ }^{3}$ Departamento de Genética Médica, Unidad Médica de Alta Especialidad, Hospital de Gineco-Obstetricia No. 4 "Luis Castelazo Ayala",

Instituto Mexicano del Seguro Social, México, D.F., México

${ }^{4}$ Laboratorio de Genómica, Genética y Bioinformática,

Hospital Infantil de México "Federico Gómez”, México, D.F., México

${ }^{5}$ Laboratorio de Medicina Regenerativa y Estudios en Cáncer,

Departamento de Posgrado, Escuela Superior de Medicina, Instituto

Politécnico Nacional, México, D.F., México

Corresponding author: J. Villa-Morales

E-mail: jusyvm@hotmail.com

Genet. Mol. Res. 14 (3): 8326-8337 (2015)

Received October 22, 2014

Accepted April 28, 2015

Published July 27, 2015

DOI http://dx.doi.org/10.4238/2015.July.27.21

ABSTRACT. Neurofibromatosis type I is an autosomal dominant disease with complete penetrance and variable age-dependent expressivity. It is caused by heterozygous mutations in neurofibromin 1 (NF1). These occur throughout the length of the gene, with no apparent 
hotspots. Even though some mutations have been found repeatedly, most have been observed only once. This, along with the variable expressivity, has made it difficult to establish genotype-phenotype correlations. Here, we report the clinical and molecular characteristics of four pediatric patients with neurofibromatosis type I. Patients were clinically examined and DNA was extracted from peripheral blood. The whole coding sequence of $N F 1$, plus flanking intronic regions, was examined by Sanger sequencing, and four frameshift mutations were identified. The mutation c.3810_3820delCATGCAGACTC was observed in a familial case. This mutation occurred within a sequence comprising two 8-bp direct repeats (GCAGACTC) separated by a CAT trinucleotide, with the deletion leading to the loss of the trinucleotide and the 8-bp repeat following it. The deletion might have occurred due to misalignment of the direct repeats during cell division. In the mutation c.5194delG, the deleted $\mathrm{G}$ is nested between two separate mononucleotide tracts (AAAGTTT), which could have played a role in creating the deletion. The other two mutations reported here are c.4076_4077insG, and c.3193 3194insA. All four mutations create premature stop codons. In three mutations, the consequence is predicted to be loss of the GAP-related, Sec14 homology, and pleckstrin homology-like domains; while in the fourth, only the latter two domains would be lost.

Key words: Neurofibromatosis type I; NF1; Frameshift mutation; Pediatric patients; Direct repeats; Homopolymeric tract

\section{INTRODUCTION}

Neurofibromatosis type I (NF1) (OMIM \#162200) has an incidence of 1 in 25003500 , and is characterized by cutaneous café-au-lait spots, skin-fold freckling, ocular Lisch nodules, neurofibromas, and optic gliomas (National Institutes of Health, 1988). Additional features, seen in a smaller proportion of affected individuals, include bone disorders such as sphenoid dysplasia and cortical thinning of the long bones, facial dysmorphism, and learning disability. Neurofibromatosis type I has age-dependent variable expressivity, with some features being apparent at a very early age (e.g., café-au-lait spots and optic glioma), and others appearing at a later age (e.g., an increase in the number of café-au-lait spots and Lisch nodules, and an increased prevalence of scoliosis). Affected individuals are at an increased risk of mortality due to malignant peripheral nerve sheath tumors and optic gliomas (Duong et al., 2011; Evans et al., 2011).

Neurofibromatosis type I is an autosomal dominant disease with complete penetrance, caused by mutations in neurofibromin 1 (NF1), a gene located on the long arm of chromosome 17q11.2 (Viskochil et al., 1990; Wallace et al., 1990). The gene has 60 exons, some of which are present only in specific transcripts due to alternative splicing. NF1 is expressed in most tissues, with a higher level of expression observed in the brain, spinal cord, and the peripheral nervous system (Daston et al., 1992). The canonical protein comprises 2818 amino acid residues and acts as a tumor suppressor (Cichowski and Jacks, 2001). Its GAP-related 
domain promotes the conversion of active Ras-GTP into inactive Ras-GDP, thus contributing to the regulation of cell growth and proliferation. When these functions are disrupted, such as through mutations and deletions, tumor formation might ensue (Serra et al., 2000, 2001). Consistent with this, individuals with neurofibromatosis type I are prone to tumor formation. When a second-hit mutation occurs in the $N F 1$ gene, cutaneous neurofibromas and plexiform neurofibromas arise. Although these are both benign tumors, plexiform neurofibromas may give rise to malignant peripheral nerve sheath tumors through the accumulation of mutations in other genes such as CDKN2A, CDKN2B, and TP53 (Menon et al., 1990; Beert et al., 2011). In addition to the GAP-related domain, neurofibromatosis type I has several other less wellstudied domains including the Sec14 homology domain and the pleckstrin homology (PH)like domain (D'Angelo et al., 2006).

Individuals with neurofibromatosis type I are heterozygous for mutations in NF1. Roughly half of all mutations arise de novo and, in fact, $N F 1$ has one of the highest mutation rates reported in humans. A large number of mutations have been reported [Human Gene Mutation Database (http://www.hgmd.cf.ac.uk/ac/) and the Leiden Open Variation Database (http://www.lovd.nl/NF1)]. Most of the mutations that have been identified are intragenic point mutations or small indels (Sabbagh et al., 2013), with only 5-10\% individuals harboring a deletion of the whole gene plus its flanking regions (Pasmant et al., 2010). Individuals carrying these large deletions tend to present with a more severe phenotype (Mautner et al., 2010).

Here, we present the clinical characteristics of four pediatric patients, together with the sequence analysis of their NF1 genes including both the coding sequence and the flanking intronic regions.

\section{MATERIAL AND METHODS}

\section{Patients and samples}

Pediatric patients with clinical features of neurofibromatosis type I were referred to the Clinical Genetics Unit of Hospital de Pediatría "Dr. Silvestre Frenk Freund", Centro Médico Nacional Siglo XXI, Instituto Mexicano del Seguro Social, in Mexico City, where they were invited to participate in the study "Association of NF1 mutations, neurofibromin expression, RAS activation state, and clinical expressivity in patients, and their families, having neurofibromatosis type I". This project was performed under approval of the institution's Research and Ethics Committee (project registration No. R-2011-3603-34) and all procedures were performed in accordance with the Declaration of Helsinki, and following the national legislation on health research with human subjects. Blood samples were collected after obtaining informed consent.

The diagnosis of neurofibromatosis type I was made following the criteria established by the NIH Consensus Conference held in 1987 (National Institutes of Health, 1988). The presence of Lisch nodules was determined by an ophthalmologist by slit lamp examination, while the presence of optic gliomas was assessed by magnetic resonance imaging. When bone anomalies were apparent, X-ray studies were performed. In all cases, family history was established and both parents were clinically examined to determine whether the syndrome was inherited. 


\section{DNA amplification and sequencing}

DNA was extracted from peripheral blood samples using the DNeasy Blood and Tissue Kit (Qiagen, Mexico City, Mexico). All 60 exons of NF1 were amplified by polymerase chain reaction (PCR) using published primer sequences covering all exons plus their flanking intronic regions (Han et al., 2001). Amplifications were performed using $250 \mathrm{ng}$ DNA, 10 pmol of each primer and $12.5 \mu \mathrm{L} 2 \mathrm{X}$ LongAmp Taq Master Mix (NEB, Ipswich, MA, USA) in a 25$\mu \mathrm{L}$ total reaction volume. PCR products were purified with the QIAquick PCR Purification Kit (Qiagen). Sequencing was performed on $45 \mathrm{ng}$ purified product using the BigDye kit (Applied Biosystems, Carlsbad, CA, USA); sequencing reactions were performed in both directions. The sequencing products were cleaned with Centri-Sep spin columns (Applied Biosystems) before running them through a 16-capillary 3130xl Genetic Analyzer (Applied Biosystems).

\section{Sequence analysis}

Chromatograms were analyzed with Phred (version: 0.020425.c) (Ewing and Green, 1998; Ewing et al., 1998); Phrap and Cross match (version 0.990319); PolyPhred (version 6.18, April 29, 2009) (Nickerson et al., 1997; Stephens et al., 2006), and Consed (version 20.0) (Gordon et al., 1998). For comparison of the patient sequences with an NF1 reference sequence, a pseudochromatogram was generated with Sudophred (version 6.18.; April 29, 2009), using positions 17: 31,094,927 to 31,382,116 of the human genome (Ensembl release 76, GRCh38) (http://www.ensembl.org/Homo_sapiens/Info/Index). Polyphred was used to label the following features: low quality regions (according to their Phred quality score), regions that could not be aligned (usually the first bases of each sequence), polymorphisms, mutations, indels, microsatellites, and repeat sequences. To this end, the following arguments were used: -extended_genotype -flanking 15 -inav on -indel 50 -ms on -md 633333333333 -nav on -o polyphred.out -refcomp on -s /. -snp on -tag genotype. The output showing the labeled positions was visually inspected with Consed in order to accept, reject, or edit the results manually. Mutations are described following the recommendations of the Human Genome Variation Society (http://www.hgvs.org/mutnomen/) (den Dunnen and Antonarakis, 2000), using as reference the NF1 sequence obtained from Locus Reference Genomic (http://www. lrg-sequence.org/) (Dalgleish et al., 2010), which corresponds to the NCBI reference sequence NM_000267.3. The beginning and ending coordinates for this sequence are 17:31095310$313 \overline{7} 4155$ in the human genome assembly GRCh38, which is the reference sequence used by the Leiden Open Variation Database to describe mutations in NF1. The following databases were consulted to determine whether the mutations were novel: Ensembl, the Human Gene Mutation Database (1239 NF1 mutations publicly available, October 2014), and the NF1 Leiden Open Variation Database (containing 1703 NF1 unique variants, October 2014).

\section{RESULTS}

In this study, four children with neurofibromatosis type I were examined and all exons of their $N F 1$ genes, plus the flanking intronic regions, were sequenced. Table 1 summarizes the clinical findings in these four patients. Even though these patients were young, with the oldest aged 8 years and the youngest, 2 years, they all had between 2 and 5 years old of the criteria required for an neurofibromatosis type I diagnosis. All 4 had a large number of café-au-lait 
spots and skin-fold freckling, while three of the four had Lisch nodules. These three characteristics were met by almost all patients diagnosed with neurofibromatosis type I reported in the literature. Most café-au-lait spots were found in the trunk and extremities, which is consistent with standard findings reported for this disease. Optic glioma, scoliosis, and affected relatives were characteristics observed only once in our set, and each was present in a different patient. The 4 patients showed some facial dysmorphism, primarily in the form of facial asymmetry.

Table 1. Clinical features and mutation characteristics of four pediatric patients with neurofibromatosis type 1.

\begin{tabular}{|c|c|c|c|c|c|}
\hline Patient & & 1 & 2 & 3 & 4 \\
\hline Age (years) & & 5 & 5 & 8 & 2 \\
\hline Gender & & Male & Male & Male & Male \\
\hline \multicolumn{6}{|l|}{ Skin symptoms } \\
\hline \multirow{11}{*}{ Café au lait spots } & Face $(0.5-1.5 \mathrm{~cm})$ & - & 5 & 2 & 1 \\
\hline & Face $(>1.5 \mathrm{~cm})$ & - & 1 & 2 & - \\
\hline & $\operatorname{Neck}(0.5-1.5 \mathrm{~cm})$ & - & - & - & 1 \\
\hline & $\operatorname{Neck}(>1.5 \mathrm{~cm})$ & - & - & - & - \\
\hline & Trunk $(0.5-1.5 \mathrm{~cm})$ & 19 & 23 & 16 & 16 \\
\hline & Trunk $(>1.5 \mathrm{~cm})$ & 5 & 1 & 15 & 1 \\
\hline & Upper extremities $(0.5-1.5 \mathrm{~cm})$ & 3 & 6 & 7 & 3 \\
\hline & Upper extremities $(>1.5 \mathrm{~cm})$ & 2 & - & 1 & - \\
\hline & Lower extremities $(0.5-1.5 \mathrm{~cm})$ & 4 & 13 & 3 & 3 \\
\hline & Lower extremities $(>1.5 \mathrm{~cm})$ & 2 & 6 & 1 & - \\
\hline & Total & 35 & 64 & 47 & 34 \\
\hline \multirow[t]{6}{*}{ Subcutaneous neurofibromas } & Head & - & - & - & 3 \\
\hline & Neck & - & - & - & - \\
\hline & Trunk & 7 & - & - & - \\
\hline & Upper extremities & - & - & - & - \\
\hline & Lower extremities & 1 & - & - & - \\
\hline & Total & 8 & - & - & 3 \\
\hline \multirow[t]{6}{*}{ Plexiform neurofibromas } & Head & 1 & - & - & - \\
\hline & Neck & - & - & - & - \\
\hline & Trunk & - & - & - & - \\
\hline & Upper extremities & - & - & - & - \\
\hline & Lower extremities & - & - & - & - \\
\hline & Total & 1 & - & - & - \\
\hline Skin-fold freckling & & Yes & Yes & Yes & Yes \\
\hline \multirow[t]{2}{*}{ Eye symptoms } & Lisch nodules & Yes & No & Yes & Yes \\
\hline & Optic glioma & No & No & No & Yes \\
\hline Skeletal symptoms & & No & No & Scoliosis & No \\
\hline Family history & First-degree relative with NF1 & Yes & No & No & No \\
\hline \multirow[t]{3}{*}{ Other clinical features } & Facial dysmorphism & Yes & Yes & Yes & Yes \\
\hline & Learning disability & No & No & Possibly & No \\
\hline & Congenital glaucoma & Yes & No & No & No \\
\hline \multirow[t]{3}{*}{ NF1 mutation characteristics } & Mutation type & Deletion & $\begin{array}{c}\text { Insertion } \\
(1 \mathrm{bp})\end{array}$ & $\begin{array}{c}\text { Deletion } \\
\text { (1 bp) }\end{array}$ & $\begin{array}{c}\text { Insertion } \\
(1 \mathrm{bp})\end{array}$ \\
\hline & Exon containing the mutation & 28 & 30 & 37 & 24 \\
\hline & Length of truncated amino acid sequence & 1278 & 1376 & 1763 & 1087 \\
\hline
\end{tabular}

NF1 = neurofibromatosis type 1 .

Indels were observed in all four patients in a heterozygous state (Figures 1-4), all of which were predicted to lead to frameshifts and to premature stop codons.

Patient 1 was a 5-year-old boy with subcutaneous neurofibromas and the only patient showing a plexiform neurofibroma. Both parents were clinically examined, and a diagnosis of neurofibromatosis type I was made for the mother. This is the only familial case among the 4 cases reported here. The proband's mother was examined and a clinical diagnosis of neurofibromatosis type I was made. Through an interview with the mother, 
it was established that other affected relatives included a maternal aunt of the proband, his maternal grandmother, and quite possibly, his older sister. The latter was described as having café-au-lait spots. However, all affected relatives except the proband's mother declined to be examined. Other unique features in this patient were an orbital subcutaneous neurofibroma and the presence of congenital glaucoma. The mutation was an 11-bp deletion in exon 28: c.3810_3820delCATGCAGACTC (Figure 1). This deletion is expected to cause a shift in the reading frame and the appearance of a premature stop codon, leading to a predicted amino acid sequence of 1278 residues, as opposed to the normal protein with 2818 residues.

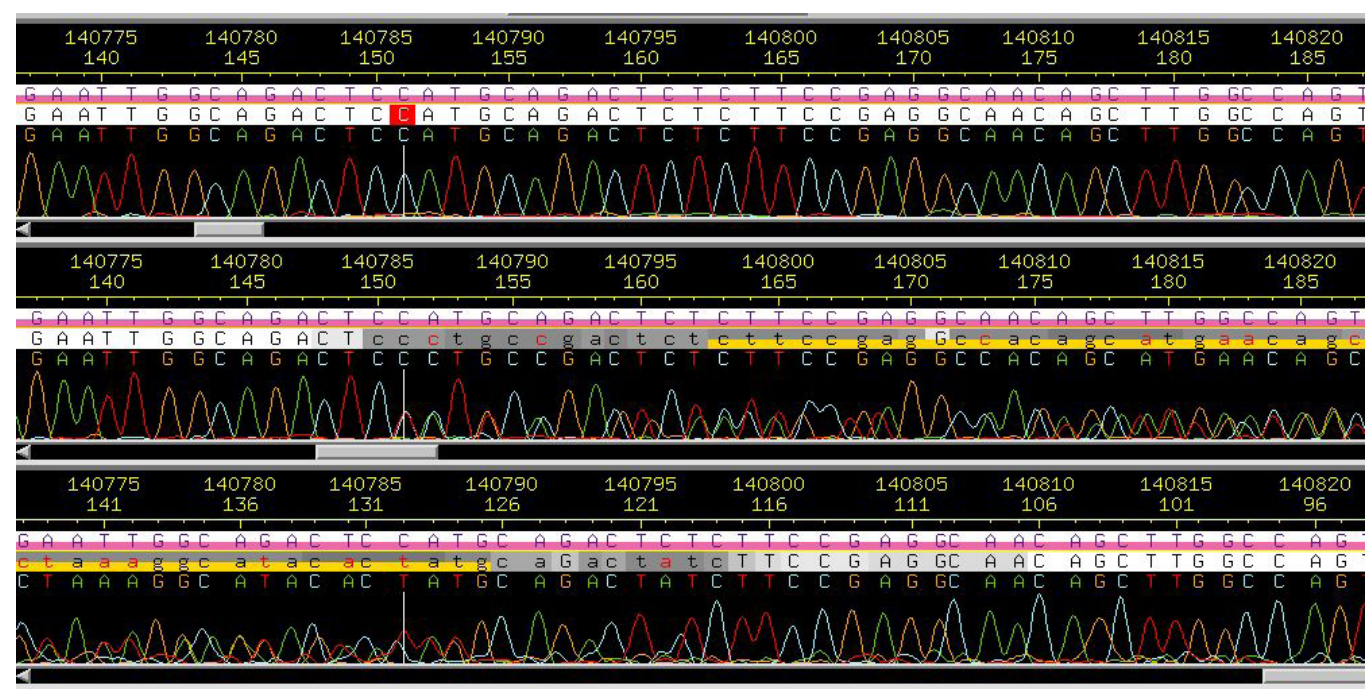

Figure 1. Case 1: eleven base pair deletion in exon 28 of NF1: c.3810_3820delCATGCAGACTC. The topmost sequence corresponds to a wild-type sample displaying the reference sequence. The pink bar under the nucleotide sequence indicates exonic sequence. The second and third chromatograms correspond to the mutated sequence (forward and reverse strands, respectively). The white vertical line indicates the starting position of the deletion.

Patient 2 also was a 5-year-old boy, who exhibited only the two most common characteristics of neurofibromatosis type I: café-au-lait spots and skin-fold freckling. This patient had no characteristics of note unrelated to neurofibromatosis type I. The identified mutation was a c.4076_4077insG in exon 30, with a premature stop codon in exon 31 leading to a predicted 1376 amino acid sequence (Figure 2).

Patient 3, an 8-year-old boy, had 3 of the most commonly observed characteristics observed in patients with neurofibromatosis type I; namely, café-au-lait spots, skin-fold freckling, and Lisch nodules. The patient had scoliosis, which is a feature that has been noted previously in a number of patients with neurofibromatosis type I. This patient repeated one school level, although this, on its own, is not enough ground for a diagnosis of learning disability, a condition that is present in a not-insignificant proportion of the general population with no association with neurofibromatosis type I. The mutation in this patient was a deletion, c.5194delG, in exon 37, which led to a predicted 1743 amino acid sequence (Figure 3). 


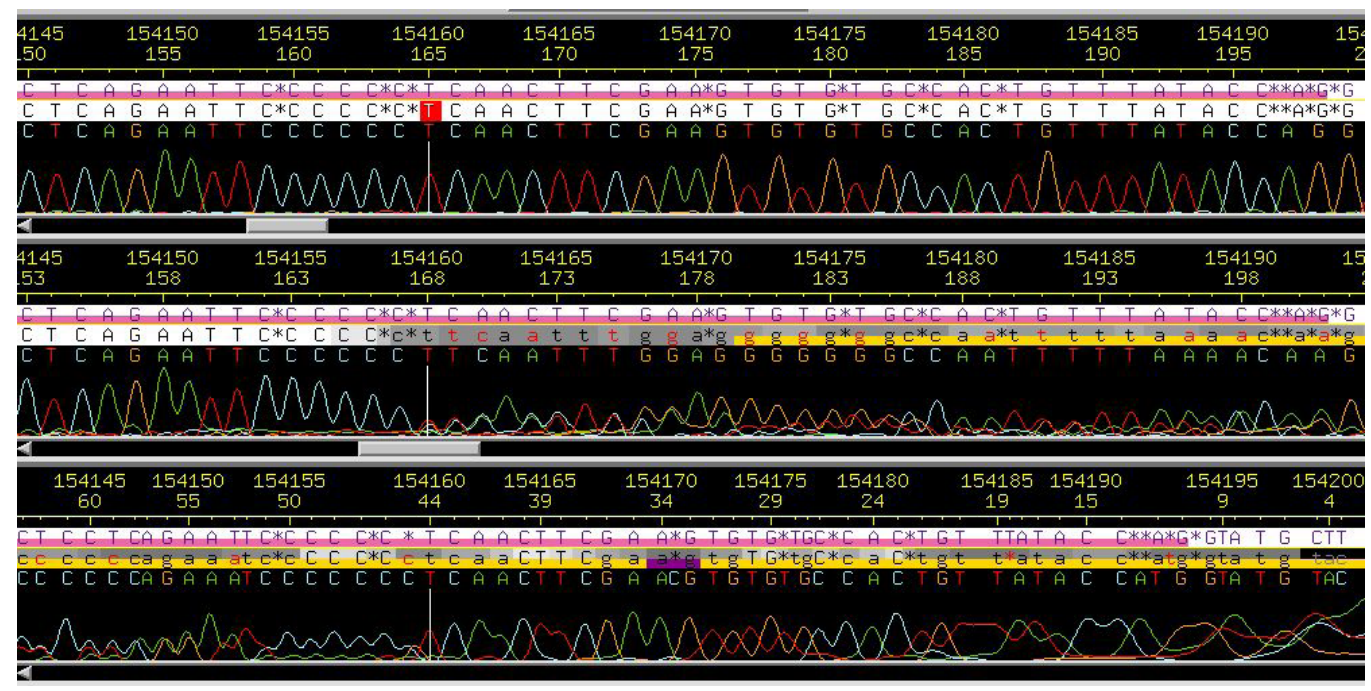

Figure 2. Case 2: insertion of a guanine in exon 30 of NF1: c.4076 4077insG. The topmost sequence corresponds to a wild-type sample displaying the reference sequence. The pink bar under the nucleotide sequence denotes the exonic sequence. The second and third chromatograms correspond to the mutated sequence (forward and reverse strands, respectively). The white vertical line indicates the position where the deletion took place.

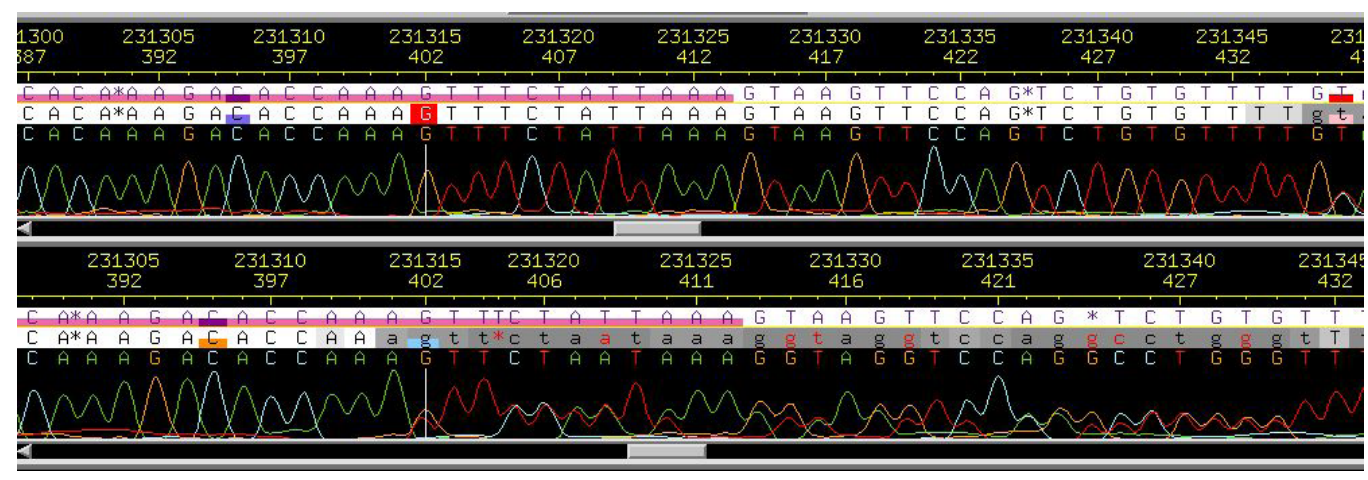

Figure 3. Case 3: deletion of a guanine in exon 37 of NF1: c.5194delG. The top chromatogram corresponds to a wild-type sample displaying the reference sequence. The pink bar under the nucleotide sequence indicates exonic sequence. The second chromatogram corresponds to the forward strand of the mutated sequence. The white vertical line indicates the position where the deletion occurred.

Finally, patient 4 presented 5 of the 7 features associated with neurofibromatosis type I, even though at age 2 years, he was the youngest of the subjects (Table 1). This patient had optic glioma. The mutation observed in exon 24 of his NF1 gene was a c.3193_3194insA (Figure 4). Given the position of this mutation, the predicted amino acid sequence, with only 1087 residues, would be the shortest of all four found in the current study. 


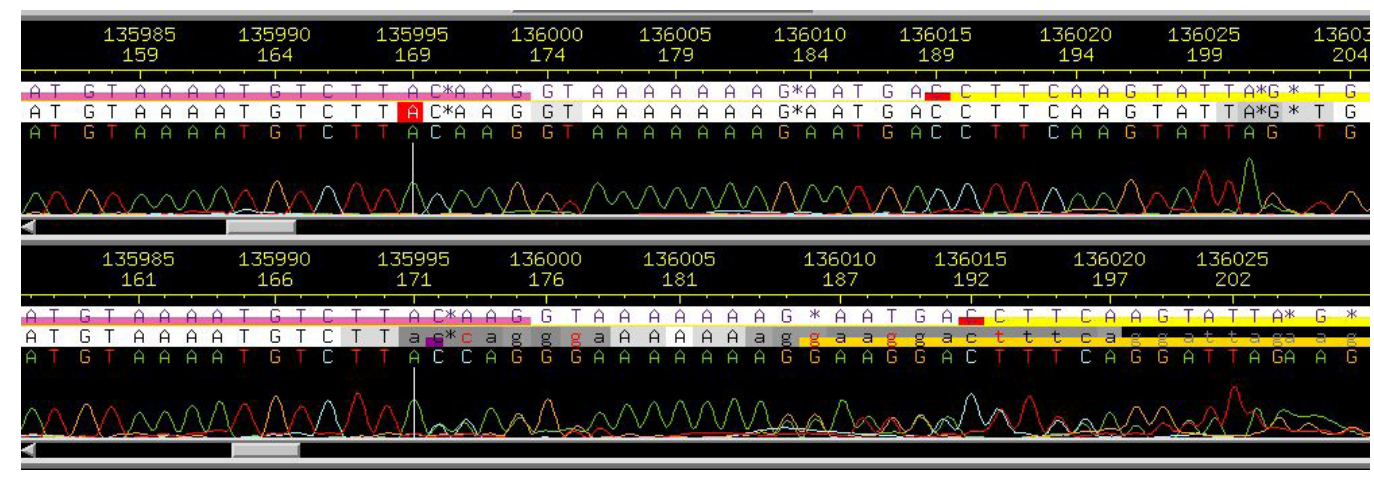

Figure 4. Case 4: insertion of an adenine in exon 24 of NF1: c.3193 3194insA. The top chromatogram corresponds to a wild-type sample displaying the reference sequence. The pink bar under the nucleotide sequence indicates exonic sequence. To its right, the red bar followed by the bright yellow bar corresponds to the reverse primer. The bottom chromatogram corresponds to the forward strand of the mutated sequence. Note that because the insertion is close to the end of the PCR product, it was not possible to get a good sequence from the reverse strand. The white vertical line indicates the position where the insertion took place.

\section{DISCUSSION}

\section{Clinical findings}

In this paper, we report the clinical and molecular findings of 4 pediatric male patients with neurofibromatosis type I; the youngest aged 2 years, and the two oldest patients aged 8 years. Neurofibromatosis type I shows age-dependent variable expressivity, with some features being apparent since birth such as café-au-lait spots, others appearing at a very early age (e.g., optic glioma), and still others during early or late adulthood (e.g., an increase in the number of neurofibromas or malignant transformation of plexiform neurofibromas). According to the NIH consensus (National Institutes of Health, 1988), for a diagnosis of neurofibromatosis type I, at least two of the seven possible criteria must be met. Given that the expressivity of neurofibromatosis type I is age dependent, some very young patients might not present with the minimum of two of the required criteria (Peltonen and Poyhonen, 2012). By the age of 8 years, however, a diagnosis of neurofibromatosis type I can be reliably made (DeBella et al., 2000). All 4 of the patients presented here met at least two NIH criteria and, in fact, the youngest patient had 5 of the 7 criteria. All 4 patients presented café-au-lait spots and skin-fold freckling, which are features present in almost all neurofibromatosis type I patients. Lisch nodules also occur in most patients, and they were present in 3 of the 4 boys examined here. One patient repeated a school year; whereas this might suggest a learning disability, this condition was not formally established with ad-hoc tests (Pride and Nort, 2012). Neurofibromatosis type I was maternally inherited in one patient, while the rest of the cases were the result of de novo mutations.

\section{Mutations}

Mutations in patients with neurofibromatosis type I have been reported across the whole gene. Even though there are no narrowly defined mutation hot-spots, some mutations 
do occur more frequently than others. For example, among inherited single base pair mutations, roughly half are nonsense mutations. Furthermore, many single base pair mutations in NF1 occur in $\mathrm{CpG}$ dinucleotides or in $\mathrm{CpNpG}$ trinucleotides as a result of the spontaneous deamination of 5-methylcytosine (Cooper and Upadhyaya, 2012). Microdeletions in mononucleotide repeats or in short tandem repeat sequences [e.g., the TGGT tetranucleotide tandem repeat (Sabbagh et al., 2013)], also seem to be relatively common, and they are thought to occur due to slippage during replication. Nonsense mutations, as well as many deletions and insertions, give rise to premature stop codons. All 4 of the patients reported here had mutations leading to frameshifts and premature stop codons. Three of the mutations have not previously been reported (c.4076_4077insG, c.5194delG, c.3193_3194insA), while the mutation c.3810 3820delCATGCAGACTC has been found once before in a patient seen in Rotterdam, The Netherlands [Leiden Open Variation Database (LOVD) DB-ID NF1_00398].

None of the mutations reported here involve mononucleotide tracts. However, in the mutation c.5194delG, the deleted $\mathrm{G}$ is in a position flanked by an A trinucleotide on one side, and by a $\mathrm{T}$ trinucleotide on the other (Figure 3 ). Whether these neighboring mononucleotide repeats could have had a role in causing the mutation is unclear. While this c.5194delG mutation has not previously been reported, a c.5191A $>$ T nonsense mutation [p.(Lys1731*); LOVD DB-ID NF1_00620] has been found in the first A of the A trinucleotide that precedes it.

As with mononucleotide tracts, tandem repeat sequences were not seen to be involved in any of the mutations reported here. However, a repeat sequence seems to have had a role in the genesis of the c.3810_3820delCATGCAGACTC mutation. As mentioned above, this mutation has also been reported in a patient seen in Rotterdam (LOVD DB-ID NF1_00398). This mutation causes a frameshift and is pathologic. Given that the exact same mutation, involving an 11-bp deletion, appeared independently in two instances, it is likely that this sequence, or the sequence within which it is embedded, facilitates the occurrence of the deletion, albeit at a very low frequency. A hint into the mechanism underlying this potential phenomenon might lie in the fact that the sequence GCAGACTCCATGCAGACTC is composed of an 8-bp direct repeat sequence (underlined bases) separated by a CAT trinucleotide. In all likelihood, these direct repeat sequences played a part in the deletion.

Although the mutation c.4076_4077insG has not been reported before, mutations in the positions flanking this insertion have been observed: c.4077delT (LOVD DB-ID NF1_00440), which causes a frameshift, and c.4078C $>$ T (LOVD DB-ID NF1_01266), which is a nonsense mutation p.(Gln1360*) (Sabbagh et al., 2013). All three mutations lead to premature stop codons, so they are expected to be pathologic.

Finally, the 3193_3194insA frameshift mutation is also novel. It is close to the end of an exon where several mutations have been reported: c.3197G>A (LOVD DB-ID NF1_00323) is a missense mutation changing the last base of the exon and has been found in three patients in The Netherlands, while mutations c. $3197+1 \mathrm{G}>\mathrm{T}$ (LOVD DB-ID NF1 01452), c. 3197+2T $>$ C (LOVD DB-ID NF1_00322) and c.3197+9dupA (LOVD DB-ID NF1_01012) occur in the flanking intronic sequence and, at least the former two, may lead to splicing errors.

\section{Genotype-phenotype correlations}

Several factors have made it difficult to find genotype-phenotype correlations in neurofibromatosis type I (Castle et al., 2003). At the molecular level, neurofibromatosis type I is characterized by its allelic heterogeneity, with mutations occurring across the whole gene 
(Human Gene Mutation Database and the NF1 Leiden Open Variation Database). Even though some NF1 mutations have been observed repeatedly, most have been found only once. At the phenotypic level, neurofibromatosis type I shows considerable variability in its expression. To further complicate matters, unrelated patients with the same mutation may present different phenotypic characteristics (Pasmant et al., 2012). Studies on patients with neurofibromatosis type 1 with varying degrees of relatedness have shown that with decreasing familial relatedness, there is also a decrease in the similarity of the neurofibromatosis type 1 phenotype (Easton et al., 1993; Szudek et al., 2002; Sabbagh et al., 2009, 2013). This argues in favor of modifier genes underlying the variable expressivity. To date, however, CDKN2B-AS1 is the only modifier gene to have been identified (Pasmant et al., 2011). This is a non-coding gene that is transcribed into a long RNA. The T allele of the rs2151280 polymorphic site within $C D K N 2 B-A S 1$ is associated with a higher number of plexiform neurofibromas. Some of the phenotypic characteristics observed in patients with neurofibromatosis type I, particularly regarding the number and distribution of the neurofibromas, might also be due to the timing and distribution of the somatic second-hit mutations required for these tumors to arise. No polymorphisms in the wild-type copy of $N F 1$ have been found to be associated with differences in the phenotype (Sabbagh et al., 2009).

Still, the fact that the NF1 protein contains several functional domains makes it conceivable that missense and some splice-site mutations occurring at different positions might lead to differences in the phenotype, since in these cases, a protein would be expected to be produced. This is in contrast with the truncation mutations where no resultant fully functional protein would be expected, due to the absence of functional domains or even because of a truncated and misfolded protein may be targeted for degradation. In this regard, a well-documented correlation between genotype and phenotype in neurofibromatosis type I corresponds to the 3-bp in-frame deletion c.2970_2972delAAT, which is associated with an absence of neurofibromas (Upadhyaya et al., 2007). Other suggested genotype-phenotype correlations include: 1) a higher proportion of non-truncating in-frame deletions in patients with neurofibromatosis type I and pulmonary stenosis, compared to patients with neurofibromatosis type I without this characteristic (Ben-Shachar et al., 2013); 2) a clustering of mutations in the $5^{\prime}$ tertile of the gene in patients with neurofibromatosis type I and optic glioma (Sharif et al., 2011); 3) a higher number of missense or missense and splice-site mutations in patients with neurofibromatosis type I and spinal neurofibromas (Upadhyaya et al., 2009); and 4) a possible association between splice-site mutations and malignant peripheral nerve sheath tumors or central nervous system gliomas in patients with neurofibromatosis type I, compared to patients with neurofibromatosis type I carrying other mutation types (Alkindy et al., 2012). A genotype-phenotype correlation has also been observed in patients with a deletion of the entire NF1 gene plus adjacent genes (Kayes et al., 1992; Kluwe et al., 2004; Mautner et al., 2010; Pasmant et al., 2010). These patients show a more severe phenotype, possibly due to the effect of the deletion of several genes along with $N F 1$.

All 4 mutations reported here cause frameshifts and lead to premature stop codons. It is possible that the resulting transcripts are degraded by nonsense-mediated decay; however, even if the respective proteins were to be produced, and if they were to escape degradation triggered by misfolding, their functions would be severely compromised. In three of them, most, or all, of the GAP-related domain would be missing, along with the residues following it, while in the fourth polypeptide sequence this domain would be present, but the Sec14 
homology domain and the neighboring PH-like domain would be absent. Therefore, all 4 of the patients reported here are expected to be haploinsufficient for $N F 1$, and their differing phenotypes are expected to be the result of as yet unknown modifier genes.

\section{Conflicts of interest}

The authors declare no conflict of interest

\section{ACKNOWLEDGMENTS}

Research supported by a Health Research Fund grant (project \#FIS/IMSS/PROT/ G12/1114) from Instituto Mexicano del Seguro Social, México. The first author is grateful to IMSS and the Hospital Infantil de México "Federico Gómez" for the support provided in order to undertake this piece of work, as part of the requirements towards a doctoral degree. Invaluable help in evaluating the patients was provided by Maria Antonieta Araujo-Solis, M.D. and Juan Carlos Huicochea-Montiel, M.D., both affiliated to the Clinical Genetics Unit of Hospital de Pediatría "Dr. Silvestre Frenk Freund", Centro Médico Nacional Siglo XXI, IMSS. Sequencing would not have been possible without the support of M. en C. Alejandro Monsalvo Reyes, UBIPRO, FES Iztacala, UNAM. We are also grateful to Douglas C. Nance (Instituto de Geriatría, México) for his assistance with the English language.

\section{REFERENCES}

Alkindy A, Chuzhanova N, Kini U, Cooper DN, et al. (2012). Genotype-phenotype associations in neurofibromatosis type 1 (NF1): an increased risk of tumor complications in patients with NF1 splice-site mutations? Hum. Genomics 6: 12.

Beert E, Brems H, Daniëls B, De Wever I, et al. (2011). Atypical neurofibromas in neurofibromatosis type 1 are premalignant tumors. Genes Chromosomes Cancer 50: 1021-1032.

Ben-Shachar S, Constantini S, Hallevi H, Sach EK, et al. (2013). Increased rate of missense/in-frame mutations in individuals with NF1-related pulmonary stenosis: a novel genotype-phenotype correlation. Eur. J. Hum. Genet. 21: 535-539.

Castle B, Baser ME, Huson SM, Cooper DN, et al. (2003). Evaluation of genotype-phenotype correlations in neurofibromatosis type 1. J. Med. Genet. 40: e109.

Cichowski K and Jacks T (2001). NF1 tumor suppressor gene function: narrowing the GAP. Cell 104: 593-604.

Cooper DN and Upadhyaya M (2012) The germline mutational spectrum in neurofibromatosis type 1 and genotypephenotype correlations. In: Neurofibromatosis Type I. (Upadhyaya M and Cooper DN, eds.). Springer-Verlag, Berlin, 115-134.

D'Angelo I, Welti S, Bonneau F and Scheffzek K (2006). A novel bipartite phospholipid-binding module in the neurofibromatosis type 1 protein. EMBO Rep. 7: 174-179.

Dalgleish R, Flicek P, Cunningham F, Astashyn A, et al. (2010). Locus Reference Genomic sequences: an improved basis for describing human DNA variants. Genome Med. 2: 24.

Daston MM, Scrable H, Nordlund M, Sturbaum AK, et al. (1992). The protein product of the neurofibromatosis type 1 gene is expressed at highest abundance in neurons, Schwann cells, and oligodendrocytes. Neuron 8: 415-428.

DeBella K, Szudek J and Friedman JM (2000). Use of the National Institutes of Health criteria for diagnosis of neurofibromatosis 1 in children. Pediatrics 105: 608-614.

den Dunnen JT and Antonarakis SE (2000). Mutation nomenclature extensions and suggestions to describe complex mutations: a discussion. Hum. Mutat. 15: 7-12.

Duong TA, Sbidian E, Valeyrie-Allanore L, Vialette C, et al. (2011). Mortality associated with neurofibromatosis 1: a cohort study of 1895 patients in 1980-2006 in France. Orphanet. J. Rare Dis. 6: 18.

Easton DF, Ponder MA, Huson SM and Ponder BA (1993). An analysis of variation in expression of neurofibromatosis (NF) type 1 (NF1): evidence for modifying genes. Am. J. Hum. Genet. 53: 305-313.

Evans DG, O’Hara C, Wilding A, Ingham SL, et al. (2011). Mortality in neurofibromatosis 1: in North West England: an 
assessment of actuarial survival in a region of the UK since 1989. Eur. J. Hum. Genet. 19: 1187-1191.

Ewing B and Green P (1998). Base-calling of automated sequencer traces using phred. II. Error probabilities. Genome Res. 8: 186-194.

Ewing B, Hillier L, Wendl MC and Green P (1998). Base-calling of automated sequencer traces using phred. I. Accuracy assessment. Genome Res. 8: 175-185.

Gordon D, Abajian C and Green P (1998). Consed: a graphical tool for sequence finishing. Genome Res. 8: 195-202.

Han SS, Cooper DN and Upadhyaya MN (2001). Evaluation of denaturing high performance liquid chromatography (DHPLC) for the mutational analysis of the neurofibromatosis type 1 (NF1) gene. Hum. Genet. 109: 487-497.

Kayes LM, Riccardi VM, Burke W, Bennett RL, et al. (1992). Large de novo DNA deletion in a patient with sporadic neurofibromatosis 1, mental retardation, and dysmorphism. J. Med. Genet. 29: 686-690.

Kluwe L, Siebert R, Gesk S, Friedrich RE, et al. (2004). Screening 500 unselected neurofibromatosis 1 patients for deletions of the NF1 gene. Hum. Mutat. 23: 111-116.

Mautner VF, Kluwe L, Friedrich RE, Roehl AC, et al. (2010). Clinical characterisation of 29 neurofibromatosis type-1 patients with molecularly ascertained $1.4 \mathrm{Mb}$ type-1 NF1 deletions. J. Med. Genet. 47: 623-630.

Menon AG, Anderson KM, Riccardi VM, Chung RY, et al. (1990). Chromosome 17p deletions and p53 gene mutations associated with the formation of malignant neurofibrosarcomas in von Recklinghausen neurofibromatosis. Proc. Natl. Acad. Sci. U. S. A. 87: 5435-5439.

National Institutes of Health (1988). Neurofibromatosis. Conference statement. National Institutes of Health Consensus Development Conference. Arch. Neurol. 45: 575-578.

Nickerson DA, Tobe VO and Taylor SL (1997). PolyPhred: automating the detection and genotyping of single nucleotide substitutions using fluorescence-based resequencing. Nucleic Acids Res. 25: 2745-2751.

Pasmant E, Sabbagh A, Spurlock G, Laurendeau I, et al. (2010). NF1 microdeletions in neurofibromatosis type 1: from genotype to phenotype. Hum. Mutat. 31: E1506-1518.

Pasmant E, Sabbagh A, Vidaud M and Bièche I (2011). ANRIL, a long, noncoding RNA, is an unexpected major hotspot in GWAS. Faseb J. 25: 444-448.

Pasmant E, Vidaud M, Vidaud D and Wolkenstein P (2012). Neurofibromatosis type 1: from genotype to phenotype. $J$. Med. Genet. 49: 483-489.

Peltonen S and Poyhonen M (2012) Clinical diagnosis and atypical forms of NF1. In: Neurofibromatosis Type I. (Upadhyaya M and Cooper DN, eds.). Springer-Verlag, Berlin, 17-30.

Pride NA and Nort KN (2012) The cognitive profile of NF1 children: therapeutic implications. In: Neurofibromatosis Type I. (Upadhyaya M and Cooper DN, eds.). Springer-Verlag, Berlin, 55-70.

Sabbagh A, Pasmant E, Laurendeau I, Parfait B, et al. (2009). Unravelling the genetic basis of variable clinical expression in neurofibromatosis 1. Hum. Mol. Genet. 18: 2768-2778.

Sabbagh A, Pasmant E, Imbard A, Luscan A, et al. (2013). NF1 molecular characterization and neurofibromatosis type I genotype-phenotype correlation: the French experience. Hum. Mutat. 34: 1510-1518.

Serra E, Rosenbaum T, Winner U, Aledo R, et al. (2000). Schwann cells harbor the somatic $N F 1$ mutation in neurofibromas: evidence of two different Schwann cell subpopulations. Hum. Mol. Genet. 9: 3055-3064.

Serra E, Rosenbaum T, Nadal M, Winner U, et al. (2001). Mitotic recombination effects homozygosity for NF1 germline mutations in neurofibromas. Nat. Genet. 28: 294-296.

Sharif S, Upadhyaya M, Ferner R, Majounie E, et al. (2011). A molecular analysis of individuals with neurofibromatosis type 1 (NF1) and optic pathway gliomas (OPGs), and an assessment of genotype-phenotype correlations. J. Med. Genet. 48: 256-260.

Stephens M, Sloan JS, Robertson PD, Scheet P, et al. (2006). Automating sequence-based detection and genotyping of SNPs from diploid samples. Nat. Genet. 38: 375-381.

Szudek J, Joe H and Friedman JM (2002). Analysis of intrafamilial phenotypic variation in neurofibromatosis 1 (NF1). Genet. Epidemiol. 23: 150-164.

Upadhyaya M, Huson SM, Davies M, Thomas N, et al. (2007). An absence of cutaneous neurofibromas associated with a 3-bp inframe deletion in exon 17 of the NF1 gene (c.2970-2972 delAAT): evidence of a clinically significant NF1 genotype-phenotype correlation. Am. J. Hum. Genet. 80: 140-151.

Upadhyaya M, Spurlock G, Kluwe L, Chuzhanova N, et al. (2009). The spectrum of somatic and germline NF1 mutations in NF1 patients with spinal neurofibromas. Neurogenetics 10: 251-263.

Viskochil D, Buchberg AM, Xu G, Cawthon RM, et al. (1990). Deletions and a translocation interrupt a cloned gene at the neurofibromatosis type 1 locus. Cell 62: 187-192.

Wallace MR, Marchuk DA, Andersen LB, Letcher R, et al. (1990). Type 1 neurofibromatosis gene: identification of a large transcript disrupted in three NF1 patients. Science 249: 181-186. 